\title{
Comparison of the Relative Performance of Active and Passive Aerosol Collection Methods for Biological Detection
}

\author{
$\underline{\text { M. J. Testolin }}^{\text {a }}$, A. Skvortsov ${ }^{\mathrm{a}}$, A. Hill ${ }^{\mathrm{a}}$ \\ ${ }^{\mathrm{a}}$ Human Protection and Performance Division, Defence Science and Technology Organisation \\ 506 Lorimer Street, Fishermans Bend, Victoria, 3207 \\ Email: Matthew.Testolin@dsto.defence.gov.au
}

\begin{abstract}
The ability to reliably detect hazardous airborne biological materials is an inherently difficult and important problem for Defence (i.e., force protection), national security (i.e., detection of anthropogenic and natural hazards, evaluation of risks for first responders) and for ecological monitoring (i.e., air pollution). There are a multitude of approaches to the problem of biological detection, all of which differ in sophistication, sensitivity, efficiency, response time, deployment feasibility and cost. A key challenge of providing sound scientific advice on a prototype detection system is the ability to evaluate against these criteria in order to select the optimal solution for the operational requirements. An example of such a study is presented in the current paper.
\end{abstract}

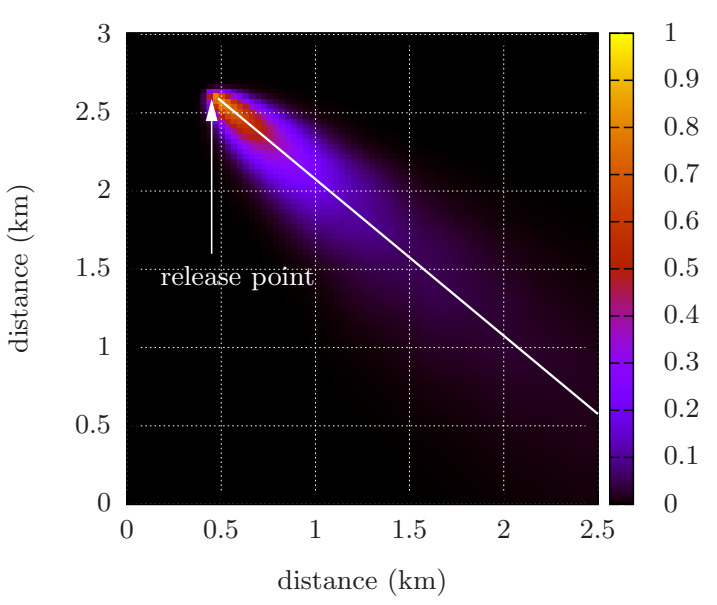

(a) Vignette four release impact (probability of infection)

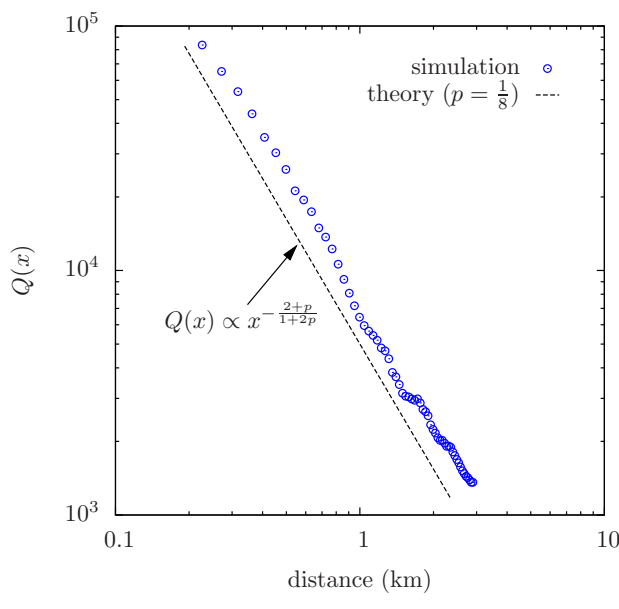

(b) Comparison between theory and simulation

Figure 1. (a) Simulation results of the release impact (vignette four, atmospheric stability condition neutral). Shown in (b) is a comparison of the theoretical scaling of active sample collection (Eq. 11) to simulation results for the release in (a) along the indicated trajectory.

Aerosol sample collection is a necessary step for many airborne biological detection systems. In these detection systems, detection is only realised when collection is combined with identification. Aerosol collection can be conditionally subdivided into two major groups: passive and active. Passive methods usually include a collection area onto which hazardous material can deposit via turbulent diffusion. Active methods rely on an active (or forced) air collection, whereby a significantly larger volume of air is sampled by a driven infiltration process. As such, active sampling systems are relatively faster and more efficient but much more costly, difficult to deploy and less scalable than passive systems.

In this paper we compare the performance of active and passive methods of aerosol collection. We demonstrate how a release of hazardous material can be modelled as a tracer puff that evolves downstream in accordance with the analytical framework of turbulent dispersion, and incorporates the basic meteorological conditions of the atmospheric boundary layer. We propose a simple physics based model for active and passive aerosol collection where the sampling time is constrained by the travelling time of the hazardous plume over the sampling point. We employ these models to estimate and compare relevant parameters of active and passive systems of aerosol collection analytically. Drawing on the simulation results from other DSTO work which examined the effects of feasible biological releases into the environment, we can validate some of these trends numerically.

Keywords: Biological detection, Aerosol collection, Active, Passive 


\section{INTRODUCTION}

The ability to reliably detect hazardous airborne biological materials is an inherently difficult and important problem for Defence, for national security and for ecological monitoring. Many factors make biological detection such a difficult problem, including that the typical size range of hazardous biological particles is between $1-10 \mu \mathrm{m}$ [see Jonsson et al. (2008)]; it is difficult to discriminate hazardous biological materials from the naturally occurring background; and effects from exposure are delayed. No single detection system is capable of reliably detecting all materials of interest, so many approaches exist, including stand-off spectroscopic techniques [Hemmer et al. (2011)], novel electrical detection [Cui et al. (2001)], detection using optical microcavities [Vollmer and Arnold (2008)] and those involving active or passive sample collection.

A key challenge for scientists working in the biological detection field is the ability to provide scientific advice on emerging approaches. In this paper we present the results of a preliminary study looking at the feasibility of using patches on soldiers for passive aerosol collection and ultimately biological detection. We compare this approach to active collection methods which are typically much more costly, difficult to deploy, provide no indication of individual exposure but are capable of sampling a large volume of air, rather than relying on particle deposition for aerosol collection.

After briefly outlining the active and passive collection methodologies, we present a simple physics based model of aerosol collection in both regimes, drawing out some key analytical trends. We quantify the relative performance further by simulating feasible biological releases, drawing on previous Defence Science and Technology Organisation (DSTO) work to provide the context. Finally the feasibility of the passive patch system and some possible concepts of use are discussed.

\section{Biological Aerosol Collection}

This study considers two airborne biological collection sample methods - active and passive. Biological sample collection is a necessary first step in the process of many biological detection systems. Neither sample collection method provides biological identification, rather they rely on other techniques such as Polymerase Chain Reaction (PCR) [Espy et al. (2006)] to achieve this. In this section we provide a brief overview of both sampling regimes, including assumptions. To judge the performance of each collection regime we carry our analysis through to biological identification by incorporating PCR analysis with sample collection. For the sake of simplicity we assume that everything collected is able to be analysed by PCR. Detection thresholds for accurate and reliable PCR identification of the agents considered in this study were based on advice provided by DSTO subject matter experts.

\subsection{Active Aerosol Collection}

Active collection methods have an active, or forced, air-sampling. As a result, active methods can draw in and sample large volumes of air to capture material. They are relatively fast and adaptable, but are much more difficult to deploy, less scalable and are more costly than passive systems.

In section 4.1 we outline our simple active sampling model, which takes time series concentration data and estimates active aerosol collection. Our model has two parameters, sampling rate $V_{s}$ and collection efficiency $\epsilon$. There are many candidate active systems, with sampling capacities ranging from $0.05-$ $60 \mathrm{~L} \mathrm{~s}^{-1}$ [CBRNe World, (2010)], however we model a generic sampler with $V_{s}=12 \mathrm{~L} \mathrm{~s}^{-1}$. We assume a relatively conservative collection efficiency of $10 \%$ [King et al. (2009)].

\subsection{Passive Aerosol Collection}

Passive collection methods rely on a collection surface (i.e. absorbing patch, Petri dish) to which material can attach driven by classical diffusion. Passive collection methods are inexpensive, straightforward to deploy, very scalable (it is not practically difficult to increase the number of absorbing patches), but much less efficient and relatively slow when compared to active sampling because they involve some post-deployment sample preparation steps.

For our simulations we take our collection surface to be a square patch of area $S_{0}=100 \mathrm{~cm}^{2}$. The patch could be attached to the clothing of a soldier or a worker who operates in an environment to be monitored for hazardous biological materials. We assume that any material deposited on the patch is used 
for identification without loss during the sample preparation phase. In reality this would not occur.

\section{THEORETICAL FRAMEWORK}

In order to provide a consistent comparison of the relative performance of active and passive aerosol collection methods we develop a simple, yet scientifically rigorous model of these processes. Our framework is based on ideas of scaling laws and self-similarity explored in Mikkelsen et al. (2002). Let us assume that at $t=0$ an amount $m_{0}$ of aerosol has been released. Parameter $m_{0}$ can be expressed in terms of the initial concentration $C_{0}$ and initial volume $V_{0}$ of the release $\left[V_{0}=(4 / 3) \pi R_{0}^{3}, R_{0}\right.$ is the initial radius of the biological plume].

For the sake of simplicity we assume that the wind can be characterised by a power-law profile [see Pasquill and Smith (1983) and references therein]

$$
U(z)=a v_{*}\left(\frac{z}{z_{0}}\right)^{p},
$$

where $v_{*}$ is the friction velocity (scale of fluctuation velocity), $z$ is the distance from the ground, $z_{0}$ is the surface roughness. Parameters $a, p$ depend on meteorological conditions, $p \in[0,1]$, with $p \approx 1 / 7$ for the stable turbulent boundary layer, as discussed in Pasquill and Smith (1983). The wind condition is usually characterised in terms of the so-called reference velocity $U_{H}=U(H)$, i.e., the mean velocity at a given height $H$ (usually $H=10 \mathrm{~m}$ ). At $z=H$ it is straightforward to see that

$$
v_{*}=\frac{1}{a}\left(\frac{z_{0}}{H}\right)^{p} U_{H}=b U_{H},
$$

so $v_{*}$ and $U_{H}$ can be used interchangeably since $b$ is constant. In the proposed framework $U_{H}$ may be viewed as an estimation for the velocity of the puff centroid displacement.

After the release, the bio-aerosol puff begins to dilute since the radius of the puff $R(t)$ increases, driven by turbulent dispersion. To characterise the evolution of $R(t)$ we can employ the ballistic model as in Mikkelsen et al. (2002)

$$
R(t) \approx R_{0}+v_{*} t
$$

Neglecting deposition due to gravitational settling, which for biological particles in the 1-10 $\mu$ m size range occurs on time scales much longer than of concern, the mean concentration in the puff decays according to

$$
C(t) \approx C_{0}\left[\frac{R_{0}}{R(t)}\right]^{3} .
$$

A simple estimate for the time taken for the puff to travel over a sampling point is $T \approx R(t) / U_{H}$, which enables us to derive the total amount of tracer $Q(t)$, captured by a detection system

$$
Q(t)=J(t) T \approx J(t) \frac{R(t)}{U_{H}}
$$

where $J(t)$ is the particle flux (mass of particles per unit time). The estimations for $J(t)$ are straightforward, that is, for the active system

$$
J(t)=V_{s} C(t),
$$

where $V_{s}$ is the air sampling rate, while for the passive system

$$
J(t)=v_{d} S C(t),
$$

where $v_{d}$ is the deposition velocity of the particles (particle size dependent), $S$ is the surface area of the collection system, (we will generalise this later to patches on the individual).

The values for parameters $v_{d}, V_{s}$ are well known: $v_{d} \approx 0.1-1 \mathrm{~cm} \mathrm{~s}^{-1}$ [for various meteorological conditions and particle sizes about $1 \mu \mathrm{m}$, see Jonsson et al. (2008)], $V_{s} \approx 0.05-60 \mathrm{~L} \mathrm{~s}^{-1}$ depending 
on the active system of interest. Rigorous estimates for the performance of active and passive sampling systems can be derived from Eqns. 3-4 and 5-7. It can be shown that $Q(t)$ decays with time,

$$
Q(t) \propto t^{-2}
$$

or, translated to the downstream distance from the release, $Q(x) \propto x^{-2}$, since $x=U_{H} t$. For an agent and detection system of interest these estimates can be used to provide a threshold condition (in terms of distance or time), outside which the deployment of the system is unjustified. Finally, active and passive systems can be used interchangeably to capture the same amount of material provided their parameters meet the following condition $S \approx V_{s} / v_{d}$. It is worth mentioning that since for the small particles $v_{d}$ is quite low, this condition can lead to very high values for the capturing area $S$ that can be impractical to implement. By considering collection patches of area $S_{0}$ on the individual, we exploit the scalable nature of passive systems by increasing the number of patches $N$, such that $S=N S_{0}$.

The reader will note that the regime $Q(t) \propto t^{-2}$ does not depend on meteorological conditions (parameter $p$ in Eq. 1). This asymptote arises from overly simplified assumptions (in particular requiring $U_{H}$ to be constant). In reality, as the puff expands there are regions of high velocity flow resulting in accelerated displacement such that,

$$
U_{H}(t) \propto t^{\frac{p}{1+p}}
$$

see Yee and Skvortsov (2011). This leads to improved estimates for $Q(t), Q(x)$, which depend on meteorological conditions

$$
Q(t) \propto t^{-\frac{2+p}{1+p}}
$$

and

$$
Q(x) \propto x^{-\frac{2+p}{1+2 p}}
$$

For $p=0$ (neutral conditions) both estimates reduce to our previous results (see Eq. 8 for example). In Fig. 1(b) simulation results (from vignette four - see Sec. 4 below) are compared to this scaling law for active sampling along the trajectory indicated in Fig. 1(a), with good agreement.

\section{Numerical Simulation}

To validate these trends an analysis of both active and passive collection methods was conducted using the Chemical, Biological and Radiological Virtual Battlespace (CBRVB), a sophisticated hazard modelling software tool developed by the UK's Defence Science and Technology Laboratory (Dstl). A series of airborne biological releases (the vignettes), originally developed for other DSTO work, were modelled to provide an indication of the relative efficiencies of active and passive aerosol collection.

\subsection{Modelling and Simulation}

The CBRVB allows specific Chemical, Biological and Radiological (CBR) releases to be overlaid on representations of military operations. The CBRVB was used to model the transport and dispersion of airborne biological releases. Each vignette was modelled in a gridded $4 \mathrm{~km} \times 4 \mathrm{~km}$ spatial domain $(32 \mathrm{~m} \times$ $32 \mathrm{~m}$ grid cell) for a careful selection of meteorological conditions (as discussed below). The output of simulations was time series of deposition and concentration data over the domain. A comparison of the active and passive collection efficiencies was conducted over the entire spatial domain by comparing for each grid cell the amount of deposited material on a small patch of area $S_{0}$ to that which was drawn into an active sampling unit $\left(V_{s}=12 \mathrm{~L} \mathrm{~s}^{-1}\right)$ from a passing plume.

Estimates of collection efficiency for both the active and passive collection regimes are based on static samplers located in each grid cell; neither accounts for the movement of personnel through a contaminated area or plume. The analysis surrounding the passive collection efficiency is based on ground level deposition data generated by the CBRVB model. It does not account for collection surfaces which may enhance the collection efficiency, such as fractal absorbers. Furthermore, we have not considered patch orientations relative to the ground but could extend any future studies to explore this. 
The modelled releases, or vignettes, were developed in support of other DSTO projects. For this study we have chosen four vignettes which adequately span an appropriate range of technical sophistication (agent preparation, scale of use and dissemination method) and consequence. It follows that the conclusions drawn in this study must be done within the context provided by these four vignettes.

Dispersion Model. The CBRVB models the transport and dispersion of airborne material using the Second-order Closure Integrated Puff (SCIPUFF) model [as described in Sykes et al. (1986)]. The fidelity of output from the SCIPUFF model is dependent on a number of key inputs, namely, the accuracy of the initial source term describing the release of material, meteorological information, land cover and terrain data. We employ the Hazard Prediction and Assessment Capability (HPAC) to define source terms for the releases.

Each vignette was simulated over a selection of representative day time meteorological conditions. Whilst night time releases are conducive to stable atmospheric conditions which result in larger hazard areas at ground level, an attack with a biological agent is most dangerous during daylight hours when people are outdoors in greater numbers. The most common atmospheric conditions during the day are neutral or unstable, depending on the wind speed, temperature, and levels of solar radiation. A complete description of the Pasquill-Gifford-Turner (PGT) atmospheric stability categories can be found in many references, including Mohan and Siddiqui (1998). We run simulations for both neutral and unstable conditions, and choose not to vary wind direction as it has no bearing on our results.

Active Sampler Model. Active sampling units collect material by forced infiltration of air into the unit. For the purposes of this study we have developed a simple model of active collection, which relies on time series data of the concentration profile. This data is an output from the CBRVB simulation. Noting that the concentration time series is a spatial array of concentration data at discrete time points, $\left\{t_{0}, t_{1}, t_{2}, \ldots, t_{n}\right\}$, we can define the rate of infiltration per time step as

$$
J_{\text {in }}\left(t_{i}\right)=C_{\text {ext }}\left(t_{i}\right) V_{s},
$$

where $C_{\text {ext }}\left(t_{i}\right)$ is the external concentration of the $i^{\text {th }}$ time step and $V_{s}$ is the sampling rate. We assume that a fraction $\epsilon$ of this material is collected each time step and the rest is exfiltrated such that $J_{\text {out }}\left(t_{i}\right)=$ $J_{\text {in }}\left(t_{i}\right)(1-\epsilon)$.

It is straightforward to see that the total mass of collected material, $Q(t)$, is

$$
Q(t)=\epsilon \Delta t \sum_{i=0}^{n} J_{\text {in }}\left(t_{i}\right),
$$

where $\Delta t=t_{i}-t_{i-1}$ is the time step - a constant. In this simple model we have made a number of assumptions: the sampler collects with no performance degradation; the external concentration remains constant for the duration of each time step (the material is well mixed); and anything not collected is exfiltrated and does not alter the concentration of the material outside. Whilst we could improve the model by refining any of these assumptions, for our purposes the simple active sampler model is sufficient.

\subsection{Results}

Ultimately we aim to gain insight into the effectiveness of active and passive sample collection as a necessary first step towards biological agent detection. To be of use, collected samples must contain sufficient agent to exceed biological identification thresholds. Presented in Tab. 1 are simulation results for active and passive collection performance across the selection of meteorological conditions outlined previously in Sec. 4.1. For each vignette and both collection methods we quote the percentage of the spatial domain where biological detection is possible. We assume 1, 100 and 1000 patches per grid cell for passive collection; we take $V_{s}=12 \mathrm{~L} \mathrm{~s}^{-1}$ in the active regime. The release impact is also characterised by stating the percentage of the domain exposed to a threshold dosage ${ }^{1}$ capable of causing infection in $1 \%$ of a healthy military population, so as to provide context for the collection performance results. For vignette four under neutral conditions we depict the release impact (taking the measure as probability of infection) in Fig. 1(a).

\footnotetext{
${ }^{1}$ Dosage is the time integrated concentration
} 
The numerical simulations confirm that passive collection methods are far less efficient than active methods. Relying on deposition alone may not be sufficient to guarantee biological detection for small scale releases such as those modelled here, even when considering many patches. This seems to agree with the theoretical trends postulated in Sec. 3. Furthermore for the release shown in Fig. 1(a) the simulation estimate of active sample collection is in agreement with the fitted scaling law (see Eq. 11), as shown in Fig. 1(b).

Table 1. Aerosol collection comparison by vignette assuming 1/100/1000 patches per grid cell for passive collection and $V_{s}=12 \mathrm{~L} \mathrm{~s}^{-1}$ in the active regime. The release impact is characterised as the percentage of the domain exposed to a threshold dosage. All values rounded to nearest integer.

\begin{tabular}{ccccccccc}
\hline \hline Collection & \multicolumn{2}{c}{ Vignette 1 } & \multicolumn{2}{c}{ Vignette 2 } & \multicolumn{2}{c}{ Vignette 3 } & \multicolumn{2}{c}{ Vignette 4 } \\
method & unstable & neutral & unstable & neutral & unstable & neutral & unstable & neutral \\
\hline Passive (1) & $0 \%$ & $0 \%$ & $0 \%$ & $0 \%$ & $0 \%$ & $0 \%$ & $0 \%$ & $0 \%$ \\
Passive (100) & $0 \%$ & $0 \%$ & $<1 \%$ & $<1 \%$ & $0 \%$ & $0 \%$ & $<1 \%$ & $<1 \%$ \\
Passive (1000) & $<1 \%$ & $1 \%$ & $9 \%$ & $15 \%$ & $<1 \%$ & $1 \%$ & $8 \%$ & $9 \%$ \\
Active & $37 \%$ & $33 \%$ & $38 \%$ & $28 \%$ & $36 \%$ & $29 \%$ & $34 \%$ & $23 \%$ \\
Release impact & $34 \%$ & $31 \%$ & $27 \%$ & $21 \%$ & $44 \%$ & $33 \%$ & $15 \%$ & $13 \%$ \\
\hline \hline
\end{tabular}

The analyses of the two sample collection performances are illustrated in Fig. 2 for the same release. In Fig. 2(a) we show the regions where identification is possible as a result of active sample collection, whilst Fig. 2(b) highlights the number of patches required to allow identification via passive collection. We have constrained the number of patches to be less than $N=1000,\left(S=10 \mathrm{~m}^{2}\right)$, bounded by operational considerations. In fact this number is likely to be much less.

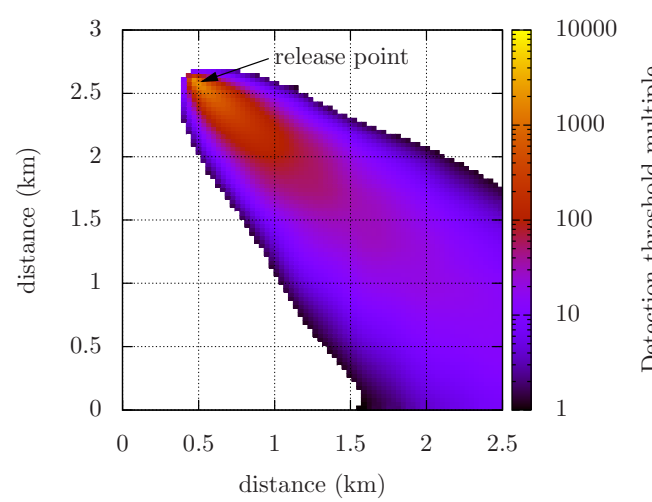

(a) Active collection

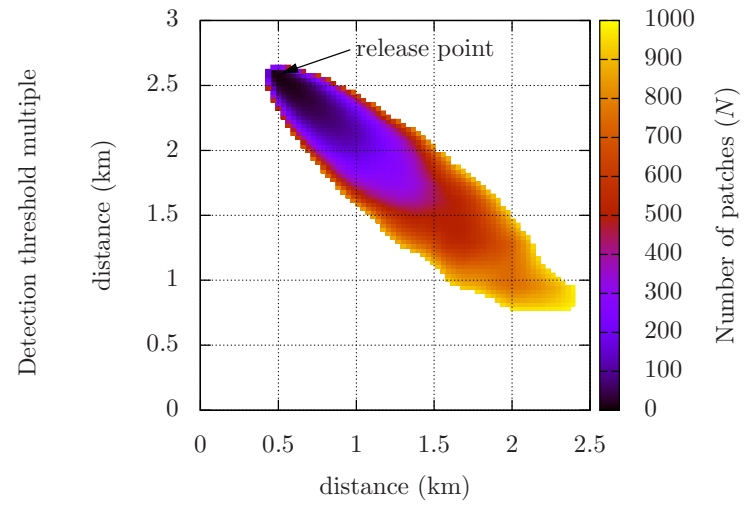

(b) Passive collection

Figure 2. Collection performance results from vignette four (neutral). In (a) we show regions of the domain where actively collected samples are above identification threshold requirements (note logarithmic colour scale), whilst in (b) we show the number $N$ of individual patches of area $S_{0}$ (up to a maximum of $N=1000$ ) required to exceed these thresholds.

\section{Discussion}

The deposition of hazardous biological materials in the $1-10 \mu \mathrm{m}$ size range has been well studied [Jonsson et al. (2008)]. For such particles these studies show that the deposition velocity, $v_{d}$, nears a minimum as a function of particle size, with typical values in the range $0.1-1 \mathrm{~cm} \mathrm{~s}^{-1}$ depending on atmospheric conditions and surface roughness. Practically this means very little deposition of biological particulates occurs on a time scale reasonable for detection - for the vignettes considered, upper bounds on time before material leaves the domain are 17 minutes (neutral), 40 minutes (unstable). This is reinforced by the theory, which exposed that the relation between patch size and $v_{d}$ is inverse. Numerical simulations revealed that in the context of the releases we considered, a single patch of area $S_{0}=100 \mathrm{~cm}^{2}$, did 
not capture enough material for successful biological detection. Exploiting the scalability of passive collection by increasing the number of patches $N$, so as to increase the collection area $\left(S=N S_{0}\right)$, would make passive collection feasible, however this poses additional challenges. Fabrication of patches from novel materials such as a fractal absorber may enhance passive collection, however this will still be dictated by $v_{d}$ and the underlying physics.

By comparison, active environmental samplers are not constrained by $v_{d}$ and are extremely efficient sample collectors, very capable of biological detection when combined with identification, as indicated by our simulation results. They are however large and costly, difficult to deploy and require a power source. In addition man portable devices are often cumbersome, meaning active samplers are more suitable as area detectors and hence provide no direct indication of individual exposure.

\section{Conclusions}

A theoretical and numerical comparison of active and passive collection has been presented. Results indicate that active collection significantly outperforms passive collection as it is not constrained by the mechanisms governing deposition of small particulates. It is however costly and difficult to deploy.

Passive collection on the other hand is highly scalable and affordable. Whilst collection from deposition on a single small patch may not be sufficient for biological detection, it is feasible that a sample prepared from many patches could be used to achieve biological identification.

\section{ACKNOWLEDGEMENTS}

The authors would like to acknowledge useful discussions with Dr Chris Woodruff, Dr Peter Gray and Dr Mick Alderton of DSTO.

\section{REFERENCES}

CBRNe World, (2010). Bio aerosol samplers. CTG 2010 (Winter), 38-39.

Cui, Y., Q. Wei, H. Park, and C. M. Lieber (2001). Nanowire nanosensors for highly sensitive and selective detection of biological and chemical species. Science 293(5533), 1289-1292.

Espy, M. J., J. R. Uhl, L. M. Sloan, S. P. Buckwalter, M. F. Jones, E. A. Vetter, J. D. C. Yao, N. L. Wengenack, J. E. Rosenblatt, I. Cockerill, F. R., and T. F. Smith (2006). Real-time PCR in clinical microbiology: Applications for routine laboratory testing. Clin. Microbiol. Rev. 19(1), 165-256.

Hemmer, P. R., R. B. Miles, P. Polynkin, T. Siebert, A. V. Sokolov, P. Sprangle, and M. O. Scully (2011). Standoff spectroscopy via remote generation of a backward-propagating laser beam. Proc. Natl. Acad. Sci. USA 108(8), 3130-3134.

Jonsson, L., E. Karlsson, and P. Jönsson (2008). Aspects of particulate dry deposition in the urban environment. J. Hazard. Mater. 153(1-2), 229-243.

King, M. D., B. F. Thien, S. Tiirikainen, and A. R. McFarland (2009). Collection characteristics of a batch-type wetted wall bioaerosol sampling cyclone. Aerobiologia 25(4), 239-247.

Mikkelsen, T., H. E. Jørgensen, M. Nielsen, and S. Ott (2002). Similarity scaling of surface-released smoke plumes. Bound.-Lay. Meteor. 105(3), 483-505.

Mohan, M. and T. A. Siddiqui (1998). Analysis of various schemes for the estimation of atmospheric stability classification. Atmos. Environ. 32(21), 3775-3781.

Pasquill, F. and F. B. Smith (1983). Atmospheric diffusion ( $3^{\text {rd }}$ ed.). Ellis Horwood, Chichester.

Sykes, R. I., W. S. Lewellen, and S. F. Parker (1986). A Gaussian plume model of atmospheric dispersion based on second-order closure. J. Clim. Appl. Meteorol. 25(3), 322-331.

Vollmer, F. and S. Arnold (2008). Whispering-gallery-mode biosensing: label-free detection down to single molecules. Nat. Methods 5(7), 591-596.

Yee, E. and A. Skvortsov (2011). Scalar fluctuations from a point source in a turbulent boundary layer. Phys. Rev. E 84(3), 036306. 